\title{
The Eighth Lake Louise Workshop on Tandem Mass Spectrometry
}

\author{
Bob Boyd \\ National Research Council, Halifax, Nova Scotia, Canada \\ Simon Gaskell \\ UMIST, Manchester, LK
}

The eighth in the annual series of workshops on tandem mass spectrometry was held from November 29 to December 2, 1995 at the Chateau Lake Louise, Alberta, Canada. Once again, the workshop attracted about 60 participants (from North America and beyond), which facilitated the active exchange of views that has characterized this series since its inception. The workshop was divided (somewhat loosely) into sessions where the focus ranged from instrumentation to applications. The influence of biological applications, however, was pervasive; even the Fundamentals session included three presentations on peptides and one on nucleotides! Don Douglas (University of British Columbia, Vancouver) opened the first session (New Techniques) with an inviled presentation on new approaches in quadrupole mass spectrometry. After a bricf discussion of high mass, high resolution instruments, he dealt at length with determinations of energy loss following collisions of multiply charged protein ions and the relationship with protein conformation. At least in some instances, satisfactory correlations with solution phase properties are observed. Terry Lee (Beckman Institute, Duarte, $\mathrm{C} \Lambda$ ) gave an extended presentation on elegant techniques developed in his laboratory for coupled microbore liquid chromatography-mass spectrometry, which included stopped-flow methods. The New Techniques session continued with short contributed presentations from Anthony Alexander (Bristol-Myers Squibb, Syracuse, NY) on B / E linked scans with a floated collision cell and from Bob Boyd (NRC, Halifax) on a new hybrid instrument based on a sector/time-of-flight (TOF) combination with the option of a magnet bypass. Arnie Falick (PerSeptive Biosystems, South San Francisco, CA) described new devclopments in biopolymer sequencing by using TOF-MS and Simon Gaskell (UMIST, Manchester, UK) reported on novel fragmentations of modified peptides and their use in screening and structural elucidation.

A short Carbohydrates session was opened by Vern Reinhold (Boston University, MA) as an invited speaker. He surveyed the very broad biological significance of glycoconjugates, particularly glycoproteins, and reviewed his laboratory's use of tandem mass spectrometry in the structural characterization of carbohydrates. Joe Banoub (Department of Fisheries and Oceans, St. Johns, Newfoundland) and Pierre Thibault
(National Research Council, Halifax, Nova Scotia) each contributed papers on the structure elucidation of complex carbohydrates and of glycoproteins, with a particular emphasis on the use of coupled capillary electrophoresis (CE) mass spectrometry.

Bill Budde (Environmental Protection Agency, Cincinnati, $\mathrm{OH}$ ) began the session on environmental applications by reviewing methods for the determination of chlorination by-products in drinking water and described recent developments in the use of tandem mass spectrometry for this purpose. This invited talk was followed by a contribution from John Headley (National Hydrology Rescarch Centre, Saskatoon, Saskatchewan) that described the mass spectrometric determination of the uptake of riverine pollutants by biofilms.

A developing tradition is for the Thursday evening session of the Lake Louise Workshop to be devoted to discussion of an emerging area in the development or application of tandem mass spectrometry. This year the discussion focused on mass spectrometric charac terization of the products of combinatorial chemistry synthetic approaches. Presentations were made by three experts in the field: Pat Griffin (Merck, Rahway, NJ), Olga Issakova (Selectide, Tucson, AZ), and Dan Kassel (CombiChem, La Jolla, CA). These speakers outlined the analytical challenges involved in assessing the success of combinatorial strategies to synthesize complete libraries and in characterizing the structures of products whose significance is established by appropriate screening strategies. Although it is clear that tandem mass spectrometry is beginning to play a role in both areas, substantial further contributions may be expected.

Friday morning was taken up with a full session on the use of ion trapping techniques in tandem mass spectrometry. Dave Laude (University of Texas, Austin, TX) presented an invited review of the developments in ion cyclotron resonance (ICR) mass spectrometry that have established this technique as a high performance tandem mass spectrometry method. In particular, he gave a critical survey of the advantages and limitations of available activation methods. Contributed presentations from John Stults (Genenlech, South San Francisco, CA) and Richard Vachet (University of North Carolina, Chapel Hill, NC) described, respectively, recent studies on the analysis of peptide 
mixtures by using matrix-assisted laser desorption ionization (MALDI) Fourier transform ICR mass spectrometry and the use of pulsed heavy gases for collisional activation of peptide ions in the quadrupole ion trap. Ray March (Trent University, Peterborough, Ontario) continued the discussion of Paul traps with a discussion of the trapping of negative ions, exemplified by the analysis of toxaphenes. A late alteration in the program resulted in the inclusion in this session of a presentation from Shannon Cornett (Bruker, Billerica, $\mathrm{MA}$ ) on the use of reflectron TOF instruments for tandem mass spectrometry analyses.

Friday afternoon was left free so that participants could take advantage of the excellent winter sports conditions or simply relax! At the workshop banquet held on Friday evening, the Chairman of the Canadian Society for Mass Spectrometry, Orval Mamer, presented an award to Alex Harrison (University of Toronto) in recognition of his many contributions to the subject of mass spectrometry and to the Canadian mass spectrometry community.

Two short sessions were held on Saturday morning. The topic of the first was designated as Fundamentals and amply illustrated the benefits of the application of basic principles to the optimization and interpretation of tandem mass spectrometric data. Keith Jenuings (University of Warwick, UK) gave an invited presentation that reviewed his laboratory's work on the mechanisms of fragmentation of peptide ions and the application of tandem mass spectrometry to the characterization of hemoglobin variants. Further studies of peptide fragmentation were described by Alex Harrison, who focused on the formation of b-type ions, and Gary Glish (University of North Carolina, Chapel Hill, NC), who described the differentiation of peptide fragment ion types on the basis of observed kinetic energy losses. In the final talk of the session, Paul Kebarle (University of Alberta, Edmonton) discussed kinetic energy thresholds for the collision-activated decomposition of mononucleotide ions.

The concluding session of the workshop covered further aspects of biological applications. An invited presentation from Steve Naylor (Mayo Clinic, Rochester, MN) reviewed his group's development of CE mass spectrometry for trace analyses, with a particular emphasis on on-line sample preconcentration techniques. The session also included short presentations from Paul D'Agostino (DRES, Suffield, Alberta) on the tandem mass spectrometry of peptides by using a hybrid sector/quadrupole instrument with moderate precursor ion resolution and from Tony Mallet (University of London, UK) on the characterization of biological markers of oxidative stress, with particular reference to hydroperoxide phospholipids.

The oral presentations were supplemented by a substantial collection of posters that were displayed throughout the workshop. The following presenters and topics comprised the workshop agenda:

Daniel Boismenu (McGill University, Montreal, Quebec), Collision-Induced Oxygen Addition to PCB Negative Ions

Shannon Cornett (Bruker, Billerica, MA), Improved $\mathrm{S} / \mathrm{N}$ and Resolution in FT MS/MS Using Quadrupolar Axialization

Simon Gaskell (UMIST, Manchester, UK), Analysis of Peptides from Solid Support by MALDI MS

Tony Gilbert (VG Organic, Manchester, UK), New Developments in Sector/ $\mathrm{IOH}$ Instruments

Jim Hancock (DRES, Suffield, Alberta), LC-ESI-MS and ESI-MS/MS of a Synthetic Peptide Reaction Mixture

Dane Karr (Teledyne, Mountain View, CA), Biomarker Analysis via Ion Trap MS/MS

John Klassen (University of Alberta, Edmonton), Metal Ion-Ligand Bonding Energies Determined by Tandem Mass Spertrometry

Qimin Li (University of Saskatoon, Saskatchewan), ES/MS/MS Analysis of the Combination Drug Azatidine with Pseudo-epinephrine; LC/MS/MS Analysis of Intact Selegiline and its Three Metabolites in Human Plasma

Kristine Swiderek (Beckman Institute, Duarte, CA), Automated Structural Characterization of Proteins and Peptides Using Electrospray LC/ MS/MS Techniques-A Presentation of Case Studies

Plans are in hand for the Ninth Lake Louise Workshop, which will be held December 4-7, 1996. 